\title{
La incidencia del self del terapeuta en el proceso terapéutico*
}

\author{
Liliana Chazenbalk** \\ Universidad de Palermo
}

Karina Mammi, Carla Nachman, Mariana Parera, Marina Reizes, Oscar Arcuschin Hoospital Alvarez

\section{Resumen}

El objetivo de este trabajo es puntuar la incidencia del estilo del terapeuta en el proceso de la psicoterapia. Definimos el concepto de estilo del terapeuta como los patrones habituales, únicos de cada persona, que se relacionan con la visión que tiene de sí mismo y del mundo, sus creencias, experiencias de vida, momento evolutivo que atraviesa, posición socioeconómica, y estilo afectivo.

Una alta similitud en el sistema de creencias entre el paciente y el terapeuta puede provocar un estancamiento en el tratamiento, como así también, una alta disonancia puede conducir al abandono del mismo.

Es fundamental que el terapeuta cuente con un espacio de supervisión y entrenamiento con el fin de abordar los siguientes items :

1- Aumentar sus conocimientos teóricos, adquirir y fortalecer un marco teórico de referencia necesario para identificar y guiar el proceso terapéutico.

2- Adquirir un amplio abanico de técnicas posibles de implementar en la psicoterapia.

3- Desarrollar habilidades internas que le permitan utilizar su experiencia personal, y desafiar las creencias disfuncionales que actúan como obstáculo en su labor.

4 Capacidad de interactuar y coordinar esfuerzos terapéuticos propios y con otros profesionales-médicos, abogados, psicopedagogos - de modo de tener una visión amplia e integral del paciente.

El poder trabajar adecuadamente estos puntos brinda al terapeuta un mayor repertorio de recursos y favorece la obtención de conductas mas flexibles y creativas.

Palabras clave: estilo del terapeuta, sistema de creencias, vínculo pacienteterapeuta.

* Trabajo presentado a las Jornadas De Psicopatolología y Salud Mental - "Encuentros y Desencuentros en Salud Mental"- Hospital Gral. De Agudos "Dr. Teodoro Alvarez".

** Master en Psicología Cognitiva - Profesora Titular de Psicología de la Facultad de Humanidades y Ciencias Sociales de la Universidad de Palermo - Coordinadora del Equipo de Familia del Hospital Gral. De Agudos "Dr . Teodoro Alvarez". 
Psicodebate 3. Psicología, Cultura y Sociedad

\section{Abstract}

The objective of this work is to stress the incidence of the therapist's style in the psychotherapeutic process. We define the concept of the therapist's style as the particular habitual patterns of each person, that are in relation with the view he has of himself and of the world, his beliefs, life experience, stage of development, socioeconomic position and emotional style.

A great similarity between the therapist' $s$ beliefs system and the patient' $s$ beliefs system can cause a block in the treatment and a big difference between them can lead the patient to abandon treatment.

It is essential for the therapist to have supervision and training to tackle the following issues:

1- To increase his theoretical knowledge, to acquire and strengthen the theoretical frame of reference needed to identify and lead the therapeutic process.

2- To acquire a wide range of possible techniques to be used in psychotherapy.

3- To develop internal abilities that allow him to use his personal experience, and challenge the dysfunctional beliefs that act like an obstacle in his work.

4 The capacity to interact and coordinate his own therapeutic efforts and with those of other professionals - doctors, lawyers, educational psychologists - to get a broader view of the patient.

This will give the therapist a larger repertoire of resources and will support the achievement of more flexible and creative behaviours.

Key words: therapist's style, beliefs' system, therapist-patient relationship. 


\section{La incidencia del self del terapeuta en el proceso terapéutico}

¿Hasta qué punto un terapeuta que está atravesando una depresión severa puede ser eficaz en su labor terapéutica?; ¿Cómo afectaría a un terapeuta judío atender a un paciente con ideas nazis?; ¿ Es posible para un terapeuta con principios y valores de justicia atender a un asesino?. En síntesis, ¿puede un terapeuta atender a un paciente con creencias muy opuestas a las suyas?.

También nos podríamos preguntar acerca de la influencia en los resultados de un tratamiento cuando el terapeuta ha padecido un trastorno similar al de su paciente y ha logrado superarlo. Pensemos, por ejemplo, el caso de un ex adicto que coordina grupos terapéuticos con personas que padecen una adicción, ¿aumenta este hecho la credibilidad de los pacientes en su terapeuta?

A continuación intentaremos vincular esta clase de interrogantes con la persona, o self del terapeuta, tomando los aportes de autores provenientes de diversas corrientes teóricas que se han ocupado de investigar dicho tema, a los fines de integrar sus ideas en la elaboración de este trabajo.

De acuerdo con la visión de Fernández Alvarez (1996) es posible concebir al "estilo" del terapeuta como los modos de ser constantes, habituales y únicos de cada sujeto que comprenden una serie de factores tales como: sus ideas, creencias, situación vital, experiencia de vida, relaciones interpersonales en general, posición socioeconómica, ámbito social, estilo afectivo, religión, procesos emotivos y cognitivos, su propia historia, visión del mundo, flexibilidad, etc.

En todos los enfoques psicoterapéuticos hay un elemento común, dado que la terapia es conducida por personas y, la relación terapéutica en tanto vínculo que se establece entre paciente y terapeuta es el vehículo para llegar a las metas trazadas.

Existe en general dentro de la comunidad terapéutica una marcada tendencia a ubicarse, adherirse y en algunos casos tomar como un dogma a determinadas corrientes psicológicas, actuando esto, como un obstáculo para generar otros posibles enfoques y/o alternativas para comprender y ayudar a los pacientes a aliviar su sufrimiento.

No cabe duda que cuanto más sólidos sean los conocimientos teóricos, mayor el abanico de técnicas que maneje el terapeuta y, cuanto mejor detecte aquello que le ocurre al paciente, podrá realizar intervenciones más precisas.

Sin embargo, debemos acentuar el papel protagónico que cobra en el proceso terapéutico el estilo personal del terapeuta, ya que como ha sido demostrado a partir de minuciosas investigaciones, tiene una alta incidencia en dicho proceso. Todo ello nos lleva a pensar que el instrumento más importante de la psicoterapia es la persona del terapeuta, como decía Satir (1985p63).

Frank (1985) a partir de sus investigaciones enumera una serie de factores que conducen al éxito en las psicoterapias:

- La capacidad que tiene el paciente de sentirse comprendido en un clima de confianza y esperanza;

- Calidad del vínculo entre paciente y terapeuta; 
Psicodebate 3. Psicología, Cultura y Sociedad

- Adquisición de informaciones nuevas, que permiten mayores posibilidades de aprendizaje;

- Activación emocional (donde la empatía, la aceptación y la autenticidad son características del terapeuta que acompañan ese proceso);

- Aumento de la sensación de dominio y autoeficacia.

Por un lado, es evidente que los pacientes tienden a obtener mayores resultados cuando asignan credibilidad al psicoterapeuta desde su inicio y por otro lado, es fundamental que el terapeuta pueda armonizar empáticamente, poniéndose en el lugar del paciente, en su marco de referencia, en su cultura, sus costumbres, sus creencias, sus valores, su manera de percibir el mundo, estableciendo sintonía con éste a nivel verbal y no verbal.

Asimismo, Beutler (1995) demostró a través de sus investigaciones, que la persona del terapeuta es ocho veces más influyente que su orientación teórica, y/o el uso de técnicas específicas terapéuticas.

Baringoltz (1992b) desarrolló intensamente esta temática planteando los siguientes interrogantes: ¿Qué determina que algunos pacientes despierten en los terapeutas diferentes conductas, emociones y pensamientos?; ¿Por qué los profesionales se sienten más cómodos con algunos pacientes que con otros?. Las respuestas a estas preguntas mantienen relación con el paradigma de los psicoterapeutas y su estilo personal.

A su vez cabe preguntarse si al existir una intensa concordancia en los estilos cognitivos de pacientes y terapeutas, esto pudiera producir un estancamiento en la psicoterapia.

Al respecto, Baringoltz (1992a) plantea: "consonancias significativas entre los sistemas de creencias de terapeuta y paciente, o un alto grado de complementariedad de los mismos, provoca estancamiento en los tratamientos, así también las disonancias importantes provocan falta de empatía, rechazo, irritabilidad y frecuente abandono del tratamiento".

A modo de ejemplo, un terapeuta con alta autoexigencia que atiende a un paciente con ideas perfeccionistas acerca de cómo trabajar; ¿podría provocar un estancamiento en el proceso terapéutico?. Dado que ambos tendrían una igual concepción acerca de la modalidad de cómo se debe trabajar ¿sería acaso más difícil para el terapeuta flexibilizar las ideas del paciente y generar alternativas?, o bien, ¿podría ser ésta una oportunidad que facilite al terapeuta revisar sus propias ideas y que, por lo tanto, lo induzca a crecer y a cambiar con el paciente?

En las investigaciones realizadas por Orlinsky, Grawe y Parks (1994), se encontró que en un $66 \%$ de los casos considerados, el vínculo terapéutico está fuertemente asociado al éxito de la terapia, y que la contribución del terapeuta al vínculo se relaciona con el éxito en un 53\% de los mismos. Los aspectos del terapeuta que han contribuido a la eficacia del tratamiento incluyen su habilidad para: conceptualizar el caso; elegir las estrategias de tratamiento apropiadas; e implementarlas en el momento adecuado, produciendo intervenciones asertivas en los planes de tratamiento de un modo consistente con su orientación teórica. Acentuamos la importancia que el terapeuta se sienta cómodo y seguro con el marco de referencia y las técnicas que utiliza. 
Otros estudios como el de Williams y Chambless (1990) demostraron mejores resultados en el proceso terapéutico cuando los pacientes perciben a sus terapeutas con un alto nivel de autoconfianza.

Desde la perspectiva de J. Bowlby (1989), la relación terapéutica no está solo determinada por la historia del paciente, sino que pone énfasis en la historia del terapeuta, quien deberá ser conciente de su propia contribución a la relación para poder actuar construyendo un vínculo de apego seguro. A grandes rasgos la teoría del apego parte de la premisa de una tendencia innata, propia de la naturaleza humana, a establecer lazos emocionales íntimos con otros individuos, tendencia que más tarde se organiza en tanto conducta de apego y que se mantiene y conserva a lo largo de toda la vida. El establecimiento de tales lazos emocionales apunta a la búsqueda de protección, consuelo y apoyo en otra persona a quien se supone dadora de dichos cuidados. Si bien existen múltiples combinaciones posibles entre los diferentes tipos de apego, es competencia del terapeuta poder detectarlas e introducirlas en la labor terapéutica. Cabe destacar que difícilmente consiga un desempeño exitoso, si previamente no ha atravesado la experiencia de ser él mismo el objeto de sus propias exploraciones. Con esto se está haciendo referencia a que el terapeuta tiene como tarea previa y continua la revisión de sus propias relaciones de apego, en tanto que la comunicación emocional con su paciente desempeñará un papel decisivo en la labor terapéutica de reestructuración de los modelos operantes del paciente. Por consiguiente, observemos que ambos aspectos, personales y teórico-técnicos, deben integrarse en vistas de configurarse como condiciones de posibilidad para el trabajo terapéutico.

Desde el enfoque cognitivo, Beck (1983) plantea la importancia de un vínculo de aceptación, empatía y autenticidad. Beck define a la aceptación como "la preocupación sincera y el interés por el paciente que puede contribuir a corregir determinadas distorsiones cognitivas negativas que el paciente aporta a la relación terapéutica”, y agrega que el factor determinante no es la real aceptación, sino la percepción de la aceptación que el paciente tenga de su terapeuta. Es más probable que las personas cooperen cuando sienten que sus creencias y sentimientos son comprendidos y respetados. Este autor define a la empatía como "la mejor manera que tiene el terapeuta para entrar en el mundo del paciente, ver y experimentar la vida como lo hace éste". Esto facilita la manifestación de sentimientos y cogniciones por parte del paciente y por lo tanto, favorece la colaboración terapéutica. Por último, Beck postula a la autenticidad como un elemento imprescindible en la relación terapéutica que debe ir acompañada por la capacidad de comunicarle su sinceridad al paciente. En síntesis, este autor respecto a la interacción terapéutica, enfatiza la confianza, el rapport y la colaboración.

En relación al entrenamiento del terapeuta hallamos los aportes de diferentes autores que son de interés para el enriquecimiento de la práctica profesional y que hacen posible la obtención de resultados de mayor confiabilidad a través de la misma.

El psicoanálisis fue el primer enfoque que incluyó en su entrenamiento profesional, la necesidad de un proceso de autoconocimiento de la persona del terapeuta, enfatizó la relación paciente-terapeuta como vehículo del tratamiento, estableciendo la necesidad del análisis didáctico para los terapeutas. 
Psicodebate 3. Psicología, Cultura y Sociedad

Freud (1933) teorizó acerca de la transferencia y la contratransferencia. Entiende la contratransferencia como los "sentimientos inconscientes" relacionados con los complejos neuróticos no resueltos del analista. Originalmente para Freud la solución para la contratransferencia era el análisis. En este sentido, Freud revisó el requisito del auto-análisis, como el proceso continuo de trabajo sobre sí mismos para los analistas.

Tanto desde el programa de entrenamiento en terapia familiar de Filadelfia (Harry Aponte), como aquel diseñado por Joan Winter, (ambos representantes de la perspectiva sistémica), coinciden en que un terapeuta es más efectivo cuando se utiliza a sí mismo para lograr la evolución tanto de su paciente, como de su propia persona. Satir (1985 p.3) propone tres objetivos principales: 1) Revelar al terapeuta la fuente de sus viejos conocimientos y su visión del mundo, 2) El desarrollo en el terapeuta de un conocimiento de sus padres como personas, mas allá del rol de padres, 3)Ayudar al terapeuta a desarrollar sus puntos de vista y definirse.

"La decisión de un terapeuta de focalizar su vida personal o su trabajo terapéutico varía, pero se supone que debe examinar ambos campos durante el período de dicho entrenamiento, ya que tanto la habilidad interna como la externa, así como la solvencia teórica y colaborativa son instrumentales para la creación de profesionales competentes". (Satir, 1972).

El programa de entrenamiento llamado "La persona y la práctica del terapeuta", pone énfasis en cuatro condiciones esenciales que el terapeuta clínico necesita para lograr un resultado terapéutico positivo (Winter, 1982,pag 4), las áreas son: 1) Posibilidades externas, la conducta técnica real utilizada por el terapeuta en el manejo de la terapia, 2) Las habilidades internas como la integración personal de la propia experiencia del terapeuta para llegar a ser un instrumento terapéutico útil, 3) La capacidad teórica, o la adquisición de modelos teóricos y marcos de referencia, necesarios para identificar y guiar el proceso terapéutico, 4) La capacidad de colaboración, o habilidad para coordinar los esfuerzos terapéuticos propios con los de otros profesionales o agentes, incluyendo médicos, docentes, abogados, otros terapeutas, etc.

$\mathrm{Si}$ bien todas las condiciones presentadas son fundamentales, dadas las limitaciones que tenemos en la extensión de este trabajo focalizaremos el centro de atención sobre la persona del terapeuta y la relación terapéutica, que entendemos como las variables fundamentales del proceso terapéutico.

La propuesta de Gallacher desde una. (1992b), perspectiva cognitiva, que desarrolla Sara Baringoltz, es el entrenamiento a partir de grupos de supervisión terapéutica. El entrenamiento mediante el dispositivo de grupos, favorece el despliegue de diferentes perspectivas y puntos de vista frente a la problemática presentada, alcanzando una visión amplificada y enriquecida tanto del paradigma del paciente como del terapeuta. Además, actúa como un espacio de contención y apoyo para los terapeutas favoreciendo a la relación paciente-terapeuta. Son de supervisión, porque se analiza la problemática del paciente y se elaboran estrategias para resolverla. Por último, son terapéuticos porque se analiza el sistema de creencias de los terapeutas y su interacción con el 
La incidencia del self del terapeuta en el proceso terapéutico

paciente, buscando una flexibilización de los mismos, permitiendo detectar los esquemas y las creencias disfuncionales del terapeuta que pudieran obstruir el desarrollo de la terapia.

A modo de ejemplo, una terapeuta recientemente recibida atendió a una familia cuyo paciente identificado presentaba varias dificultades en el área social. Luego de dos semanas, la madre le dijo a la psicóloga que no veía grandes cambios, y no sabía qué hacer con su hijo, para ella "estaba todo mal". Frente a este planteo la terapeuta se preguntó a sí misma: ¿Por qué no logro grandes cambios? ¿Será que no sirvo como profesional? ¿Me habré equivocado de profesión?. Frente a estos interrogantes, en un grupo de supervisión terapéutica se intentó desafiar estas distorsiones cognitivas: cuestionando la evidencia: ¿Qué te hace pensar que a partir de un sólo paciente no servís como profesional? ¿Acaso no hubo cambios? ¿Para quién son grandes y para quién pequeños?; a través de la reatribución: ¿No será que la expectativa de esa madre es muy ambiciosa? ¿No será que esta señora por esperar grandes cambios no puede ver aquellos que por pequeños no dejan de ser de importante valor?; examinando opciones alternativas: Todo reclamo de un paciente por esperar algo mejor ¿es un fracaso mío (terapeuta)?, ¿Solo a mí me ocurre esto?

Esto permitió que la terapeuta examine y analice sus distorsiones cognitivas conduciéndola a obtener una visión más amplia de la situación incidiendo ello notablemente en el desarrollo del proceso terapéutico. "El grupo de supervisión terapéutica es un camino hacia el enriquecimiento del significado personal del terapeuta, se abre un espacio reflexivo-vivencial en donde los terapeutas encuentran un lugar diferente al de las supervisiones individuales, inclusive al del propio análisis, tomando conciencia de las propias creencias disfuncionales y su conexión con emociones no reconocidas que permiten un mayor crecimiento tanto en lo profesional como en lo individual" (Baringoltz, 1992b).

Feixas y Miro señalan que la concepción del proceso terapéutico depende, en gran medida, del modelo psicoterapéutico que se adopte, las construcciones del paciente y del terapeuta van configurando significados que facilitan, obstaculizan o detienen el cambio.

Minuchin (1986 p23), desde la perspectiva sistémica, plantea que existen muchos terapeutas familiares que, a pesar de utilizar magníficas intervenciones, éstas resultan incorrectas cuando no guardan relación con la comprensión y las necesidades básicas de la familia.

Para Lambert (1989) "el psicoterapeuta es un factor importante en el proceso y resultado de la psicoterapia, la influencia del terapeuta permanece significativa aún en estudios donde los profesionales han sido seleccionados, entrenados, supervisados y monitoriados para minimizar diferencias en sus prácticas".

Para ir sintetizando, básicamente la formación requiere entrenamiento teórico práctico, siendo que la teoría constituye el marco de referencia para la comprensión de los modos singulares de procesar la información de cada paciente, y guía las operaciones para el cambio a través de diferentes técnicas. Sin embargo se considera también, que el exagerado control y dogmatismo del terapeuta perturban su flexibilidad y son predictores de resultados pobres. En cambio, una actitud más flexible y abierta se relaciona con resultados positivos en psicoterapia. 
Psicodebate 3. Psicología, Cultura y Sociedad

En consecuencia, la persona del terapeuta está involucrada en el vínculo y en la dirección del cambio, por lo tanto es necesario trabajar los materiales clínicos en supervisiones, asistir a ateneos, jornadas, congresos, etc. Baringoltz (1992c) dice "es fundamental centrarse en las características del estilo cognitivo del terapeuta que actúan en el entrecruzamiento de creencias propias con las de los pacientes".

Dado que el terapeuta, en general, asume un compromiso con la labor psicoterapéutica y su instrumento terapéutico es su propia persona, es fundamental que entonces cuente con un espacio de trabajo en equipo, donde se sienta contenido y acompañado , trabajando su persona como terapeuta y la de sus pares. Asimismo, tiene un considerable valor el hecho de contar con espacios de recreación, de descanso e implementación del humor, que producen distensión y mayor efectividad en su labor terapéutica. Finalmente es relevante que el terapeuta cuente con una formación creativa, incluyendo la observación de si mismo y de sus propias experiencias internas.

Para finalizar, por todos los interrogantes planteados anteriormente, es que hemos decidido focalizar este trabajo en la persona del terapeuta. Creemos que a pesar del gran número de investigaciones sobre el tema aún queda mucho por investigar.

\section{Referencias Bibliográficas}

Aponte, H.-Winter, J. (1986) La persona y la práctica del terapeuta, tratamiento y entrenamiento. Congreso Internacional de Terapia Sistémica - Filadelfia - U.S.A.

Baringoltz, S. (1996) Integración de aportes cognitivos a la psicoterapia. Buenos Aires: Belgrano.

Baringoltz, S. (1992a) El proceso terapéutico como construcción de los protagonistas de la situación clínica. Segundas Jornadas de Terapia Cognitiva, San Pablo, Brasil.

Baringoltz, S. (1992b) Enigmas y laberintos en el sesgo cognitivo del terapeuta. Congreso Internacional de Terapia Cognitiva, Toronto.

Baringoltz, S. (1992c) Un viaje distinto. Revista Argentina de Clínica Psicológica. I (1) Pág. 16 a 20. Buenos Aires: AIGLE.

Beck, A. (1995) Terapia Cognitiva de los trastornos de personalidad. Buenos Aires: Paidós.

Beck, A. (1983) Terapia Cognitiva de la depresión. Bilbao, España: Ed. Desclee de Brouwer.

Bolwby, J. (1989) Una base segura: Aplicaciones clínicas de una teoría del apego. Buenos Aires: Paidós

Chazenbalk, L. (1998) Psicología Cognitiva: enfoque teórico-práctico. Buenos Aires: E.C.U.A. 
La incidencia del self del terapeuta en el proceso terapéutico

Fernández Alvarez, H. (1997) Formación y entrenamiento de psicoterapeutas. Una perspectiva cognitiva. Desarrollos en psicoterapia. Cap. I, pág. 27 a 45. Buenos Aires: Ed. Belgrano.

Fernández Alvarez, H. (1996) Características del psicoterapeuta. Revista Dinámica. Año II, Vol. I, N²4, Pág. 1 a 24. Buenos Aires.

Freud, S. (1933) Nuevas Lecciones Introductorias al Psicoanálisis. Obras Completas de Sigmund Freud. Tomo III. Madrid: Ed. Biblioteca Nueva (1972).

Gallacher, P., Guilligan, P., Cejas, N. Rosenfeld, C. (1992) La interacción de las cogniciones de los pacientes y los terapeutas, interjuego o enredo?. Congreso Internacional de Terapia Cognitiva, Toronto.

López Mato y colaboradores (1997) Teoría y técnica sistémica. Psiquiatría Neoclásica. Vol. II, Pág. 133 a 149. Buenos Aires: Ananke.

Minuchin, S. (1986) Familias y terapia familiar. Buenos Aires: Gedisa.

Rodriguez Ceberio, M. y colaboradores (1995) Teoría y técnica de la psicoterapia sistémica. Buenos Aires: E.C.U.A.

Safran, J. y Zender, S. (1994) El proceso interpersonal en la Terapia Cognitiva. Buenos Aires: Paidós

Satir, V. (1985). Relaciones humanas en el núcleo familiar. 7ma. Edición, México: Ed. Pax. 
Psicodebate 3. Psicología, Cultura y Sociedad 